\title{
Melting moons make Tabby's Star murky
}

Mon. Not. R. Astron. Soc. 489, 5119-5135 (2019)

Tabby's Star (KIC 8462852), the main sequence star that exhibits aperiodic dips of varying depths and widths in its light curve, is possibly orbited by a giant swarm of comets or a group of more massive outgassing bodies (and those are amongst the least outlandish suggestions). However, Miguel Martinez and collaborators have put forward another viable idea for the source of the occluding material: vaporizing exomoons.

Exomoons could be stripped from their host exoplanet under the gravitational influence of the host star if the exoplanet's orbit happens to become sufficiently eccentric (through the Kozai-Lidov mechanism, for instance) that it passes close to the star. A suite of three- and four-body simulations run by the authors show that in $\sim 10 \%$ of cases the exomoons become detached from the exoplanet and start orbiting the star. The exoplanet itself is destroyed in a stellar collision (that may cause a brightening of the star). Icy exomoons with sufficiently small pericentres will be heated enough to vaporize their volatile content while also ejecting dust grains. The clouds of volatiles would occlude the star on short timescales, while the dust grains would gather together into a disk with non-uniform and possibly time-variable opacity, obscuring the star for longer periods. These phenomena would fit with the conclusion of Schaefer et al.

(Mon. Not. R. Astron. Soc. 481, 2235-2248; 2018), for example, that the short-duration dips and the longer-term secular dimming of the star are connected to the same physical mechanism. And indeed, the model of Martinez et al. fits several other observational constraints, but the authors do not make it clear how their proposed scenario could be observationally confirmed.

Paul Woods

Published online: 25 October 2019

https://doi.org/10.1038/s41550-019-0946-1 